\title{
Specific growth rate, colonial morphology and extracellular polysaccharides (EPS) content of Scenedesmus obliquus grown under different levels of light limitation
}

\author{
Ming $\mathrm{Li}^{1,2 *}$, $\mathrm{Li} \mathrm{Gao}^{3}$ and $\mathrm{Li} \mathrm{Lin}^{4}$ \\ ${ }^{1}$ College of Resources and Environment, Northwest A \& F University, Yangling 712100, PR China \\ 2 Key Laboratory of Plant Nutrition and the Agri-environment in Northwest China, Ministry of Agriculture, PR China \\ 3 Water Quality Performance \& Regulation, Coliban Region Water Corporation, Bendigo, Victoria 3554, Australia \\ 4 Department of Water Environment Research, Changjiang River Scientific Research Institute, Wuhan 430010, PR China
}

Received 14 February 2015; Accepted 27 October 2015

\begin{abstract}
In order to investigate the role of extracellular polysaccharide (EPS) in colony formation of Scenedesmus obliquus, the relationships among morphological characteristics, EPS content and specific growth rate of $S$. obliquus are investigated in this study. $S$. obliquus cultured under varying light intensities exhibited different specific growth rates, when the specific growth rate decreased from 0.65 to 0.40 day $^{-1}$ the cells per particle of $S$. obliquus increased from 1.2 to 2.8 ; but the cells per particle fell back to 1.2 as the specific growth rate further decreased from 0.40 to 0.14 day $^{-1}$. Moreover, a negative relationship between the specific growth rate and EPS content was found when the specific growth rate was lower than 0.4 day $^{-1}$; however, the EPS content maintained at a relatively steady state $\left(0.14-0.20 \mathrm{pg} \mathrm{cell}^{-1}\right)$ when the specific growth rate was higher than 0.4 day $^{-1}$. No significant relationship was identified between EPS content and cells per particle of $S$. obliquus. This result revealed that the increasing EPS content may not lead to colony formation of $S$. obliquus, which can provide a deeper insight into the role of EPS content in colony formation of different algae.
\end{abstract}

Key words: Extracellular polysaccharides / colony formation / Scenedesmus obliquus / specific growth rate / morphology

\section{Introduction}

Scenedesmus obliquus (Turpin) Kützing 1833, a common freshwater green algae, was reported as having a high degree of phenotypic plasticity (Lürling, 1999), which is considered as an important factor in evolution (Agrawal, 2001; Hairston et al., 2001). The unicell-colony transformation in $S$. obliquus could also affect the energy flow from algae to higher trophic levels in the freshwater ecosystems as unicellular Scenedesmus are easily harvested by grazing zooplankton (Lürling, 2006). S. obliquus can exist as unicellular morph or colonies depending on the different environmental conditions (Trainor, 1993; Lürling, 2003). Consequently, the influencing factors and mechanisms of colony formation of $S$. obliquus have gained significant research attention (Mulderij et al., 2005; Liu et al., 2010).

\footnotetext{
*Corresponding author: lileaf@163.com
}

Grazer-induced colony formation of $S$. obliquus has been well examined by various researchers (Lampert et al., 1994; Lürling, 2003; Yang et al., 2007) in the last 20 years, and the info-chemicals released by the grazers were considered as the main colony-inducing factors (Lürling and van Don, 1997). Furthermore, some abiotic factors, such as glyoxylate (Liu et al., 2010) and surfactant (Lürling, 2006; Li et al., 2013a) have also been reported to induce the colony formation of $S$. obliquus. However, the effects of some major abiotic factors (i.e., light, temperature, nutrient concentration) on colony formation of $S$. obliquus remain poorly understood.

Similar to S. obliquus, colony formation of Microcystis aeruginosa can also be induced by grazers (Yang et al., 2008). The mechanisms and influencing factors of colony formation of $M$. aeruginosa were well studied recently as colony formation plays an important role in Microcystis bloom formation. These studies could provide clues in understanding colony formation of S. obliquus. Light intensity (Li et al., 2013b), temperature and nutrient 
concentration (Yang and Kong, 2013) were able to induce colony formation of $M$. aeruginosa. To the best of our knowledge, the effects of the above factors on colony formation of $S$. obliquus have not been studied previously. Our previous research (Li et al., 2013b) demonstrated that the effects of these three major factors on colony formation of $M$. aeruginosa can be interpreted by the relationship between the colony size and specific growth rate of Microcystis. Nevertheless, colony formation of S. obliquus differs from that of $M$. aeruginosa occasionally (Yang and $\mathrm{Li}, 2007)$. Thus, understanding the relationship between colony size (can be represented by cells per particle) and specific growth rate of $S$. obliquus could provide a deeper insight into the effects of those major abiotic factors on colony formation of $S$. obliquus. Previous studies suggested that the production of extracellular polysaccharides (EPS) was significantly related to the colony formation of some alga, such as Chlorella pyrenoidosa (Yang et al., 2010) and M. aeruginosa (Liu et al., 2011). However, to the best of our knowledge there is no thorough and systematic study on the relationship between EPS content and colony size (or cells per particle) of $S$. obliquus until now, except for a preliminary study by Li et al. (2013a), suggesting a positive relationship between the cells per particle and EPS content of S. obliquus. A positive relationship between the cells per particle and the content of total polysaccharide (TPS) of $S$. obliquus has also been reported by Liu et al. (2010), which implied the potential significant relationship between EPS content and colony formation of $S$. obliquus. In addition, some materials were in the gap between the continuous trilaminar sheath and the ornamented layer (Pickett-Heaps and Staehelin, 1975). Even though, the composition of this layer was polysaccharides, whether they should be considered as EPS inducing colony formation should also be discussed.

This study was aimed at investigating the influencing factors and mechanisms of colony formation of S. obliquus. In our study S. obliquus was cultured under varying light intensities to obtain different specific growth rates, then the relationships between specific growth rates, EPS content and morphology of $S$. obliquus were analyzed.

\section{Materials and methods}

\section{Organisms}

S. obliquus was isolated from samples collected from Lake Taihu, China. It was cultured in CHU-10 medium (Chu, 1942) (more than 5 months) and existed as unicellular morph. Prior to the experiments, S. obliquus was cultured in BG-11 medium (Allen, 1968) for 2 weeks. The culture medium was changed because BG-11 medium was a common medium which was used in the experiments investigating effects of environmental factors on colony formation of S. obliquus (Liu et al., 2010; Chen et al., 2011; Li et al., 2013a, 2013b).

\section{Algae cultivation}

S. obliquus was batch cultured axenically in $150 \mathrm{~mL}$ of sterilized liquid BG-11 medium in a $250 \mathrm{~mL}$ conical flask at $25^{\circ} \mathrm{C}$ under varying light intensities $(10,15,20$, $25,30,35,40,50$ and $60 \mu \mathrm{mol}$ photons $\left.\mathrm{m}^{-2} \mathrm{~s}^{-1}\right)$. The light-dark cycle was 12:12 h. The initial cell density (cells number per unit volume culture) of $S$. obliquus was $5 \times 10^{4}$ cells $\mathrm{mL}^{-1}$. All cultures were prepared in triplicate. The flasks were shaken by hand two to three times daily to prevent the cells from clinging to the inner walls of the flasks. The experiment lasted for 9 days.

\section{Cell counting}

Cell density of $S$. obliquus was counted daily. The cells were directly counted thrice in a blood cell counting chamber using an optical microscope (Olympus CX31; Olympus Corporation) at $\times 400$ magnification, afterwards, the cell density was calculated. If the differences of these three calculated results were less than $10 \%$, then the average value of these results was used as the final cell density. Otherwise, additional counting was conducted.

At day 9, the number of cells per particle (cells number in a colony; both unicells and colonies were defined as particles in the current study) and the proportion of different particles were calculated by recording seriatim the number of particles and cells per particle. A minimum of 400 particles were calculated for each sample. The specific growth rate $(\mu)$ was calculated from exponential growth model similar to our previous study (Li et al., 2013b).

\section{Analysis of mean particle size and volume of S. obliquus}

Particle size of $S$. obliquus was directly measured using Mastersizer 2000 particle size analyzer based on the technique of laser diffraction. About $80 \mathrm{~mL}$ sample of each duplicate was used in the analysis $(N=29)$. The measuring approach has been well described in our previous study (Li et al., 2014). The median particle diameter $\mathrm{D}_{50}(50 \%$ of the total mass of the particles smaller than this size) was used as the mean particle size of $S$. obliquus. The mean particle volume was calculated using the mean particle size of $S$. obliquus via sphere volume formula. The volume per cell was also determined by dividing the mean particle volume by the number of cells per particle.

\section{EPS content analysis}

Accurate $20 \mathrm{~mL}$ sample of each duplicate was used in EPS analysis. EPS content was measured by the anthrone sulfuric acid method in triplicate and normalized by cell counts at day 9. The EPS was extracted by following the method described by Yang et al. (2008). 


\section{Statistical analysis}

Pearson correlation analysis was employed in the current study to analyze the correlation among specific growth rate, EPS content and cells per particle. The statistical analyses were performed using SPSS 10.0.

\section{Results}

\section{Specific growth rate at varying light intensities}

Figure 1 shows the specific growth rate of $S$. obliquus that was obviously affected by light intensity. The maximum and minimum specific growth rates were 0.65 and $0.14 \mathrm{day}^{-1}$ under light intensities of 60 and $10 \mu \mathrm{mol}$ photons $\mathrm{m}^{-2} \mathrm{~s}^{-1}$, respectively. High light intensity yielded a high specific growth rate of $S$. obliquus.

\section{Variation of EPS content relating to specific growth rates and cells per particle}

Figure 2 shows variation of EPS content relating to specific growth rates and cells per particle. A negative linear relationship was established between EPS content and specific growth rate when the specific growth rate was lower than $0.4 \mathrm{day}^{-1}$, however, the EPS content maintained at a relatively steady state $\left(0.14-0.20 \mathrm{pg}^{-1} \mathrm{cel}^{-1}\right)$ when the specific growth rate was higher than 0.4 day $^{-1}$. No significant relationship was found between EPS content and cells per particle. On the one hand, cells per particle was around 1 when the EPS content reached $1.4 \mathrm{pg} \mathrm{cell}^{-1}$; on the other hand, when cells per particle was more than 3.0, the EPS content was smaller than $0.2 \mathrm{pg}$ cell $^{-1}$.

\section{Morphological characteristics at different specific growth rates}

The proportion of different morphological particles of $S$. obliquus at different light intensities is illustrated in Figure 3. On the one hand, almost $80 \%$ of S. obliquus cultures existed as unicellular forms under the light intensities of 50 and $60 \mu \mathrm{mol}$ photons $\mathrm{m}^{-2} \mathrm{~s}^{-1}$; similarly, approximately $70 \%$ of $S$. obliquus occurred as unicellular morph when light intensity was smaller than $25 \mu \mathrm{mol}$ photons $\mathrm{m}^{-2} \mathrm{~s}^{-1}$. On the other hand, the percentage of unicellular S. obliquus decreased by around $40 \%$, and colonies composed of more than four cells represented approximately $40 \%$ of the S. obliquus cultures under the light intensities of 30 and $35 \mu \mathrm{mol}$ photons $\mathrm{m}^{-2} \mathrm{~s}^{-1}$.

Figure 4 shows morphological characteristics of S. obliquus at different specific growth rates. Mean particle size increased while the specific growth rate decreased from 0.65 to $0.40 \mathrm{day}^{-1}$. However, it was at steady state $(9-10 \mu \mathrm{m})$ while the specific growth rate decreased from 0.40 to $0.14 \mathrm{day}^{-1}$. Cells per particle increased from

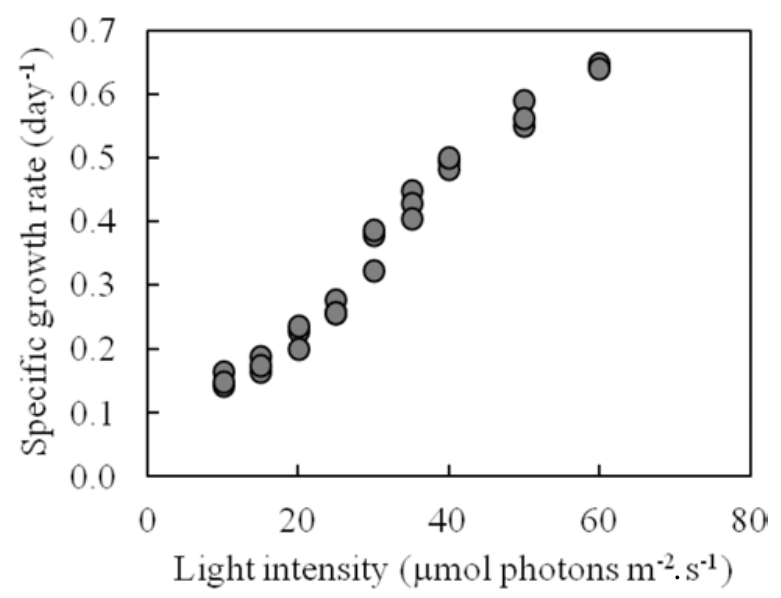

Fig. 1. Specific growth rate of $S$. obliquus at varying light intensities grown in B-11 medium.
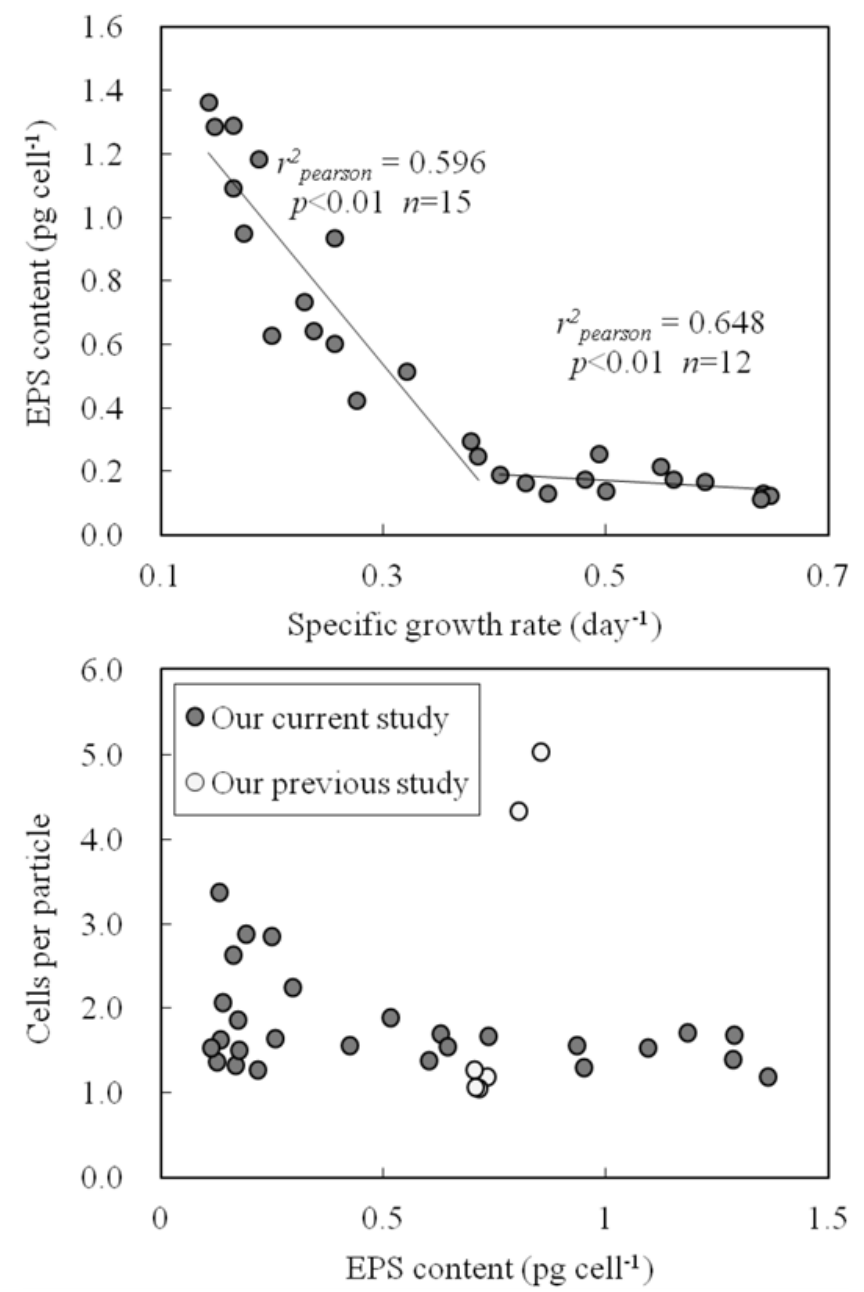

Fig. 2. Variation of EPS content relating to specific growth rates and cells per particle. The white cycle presented data induced by different concentrations of linear alkylbenzene sulfonates from our previous study (Li et al., 2013a).

1.2 to 3.0 while the specific growth rate decreased from 0.65 to $0.40 \mathrm{day}^{-1}$, but the cell per particle fell back to 1.2 as the specific growth rate further decreased from 


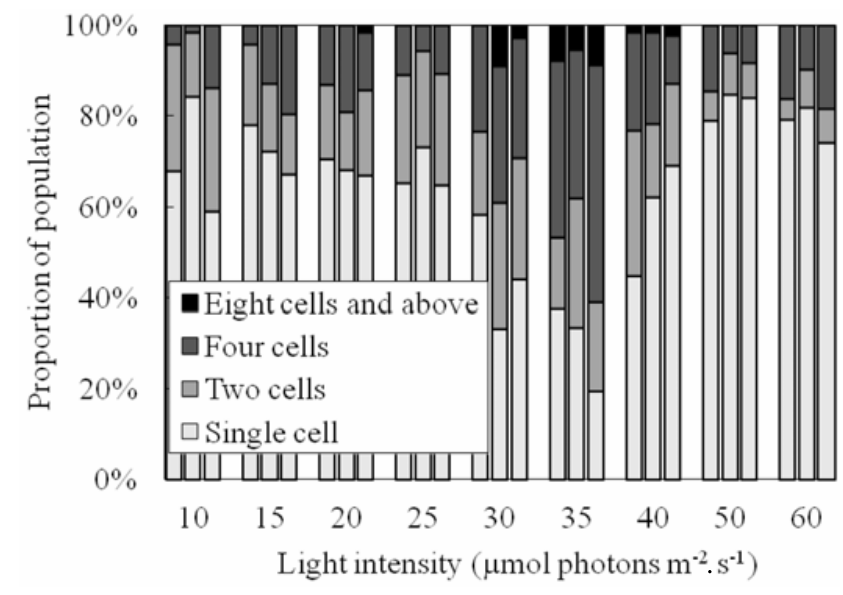

Fig. 3. The proportion of different morphological particles of S. obliquus at different light intensities.

0.40 to $0.14 \mathrm{day}^{-1}$. A significant negative relationship between the volume per cell and specific growth rate was found. Volume per cell of $S$. obliquus increased from $80 \mu \mathrm{m}^{3}$ to more than $400 \mu \mathrm{m}^{3}$ while the specific growth rate decreased from 0.65 to 0.14 day $^{-1}$.

\section{Discussion}

Li et al. (2014) reported that TPS content of Microcystis decreased along with the increase of specific growth rate because a large proportion of carbohydrates transformed to protein to maintain the high growth rate. Thus, it is hypothesized that EPS content of $S$. obliquus decreased with increasing specific growth rate induced by varying light intensities. However, our results showed that the EPS content did decrease when the specific growth rate increased from 0.14 to $0.40 \mathrm{day}^{-1}$ but it maintained at a steady state when the specific growth rate was higher than 0.4 day $^{-1}$.

Similarly, contrary to our assumption based on the previous study (Li et al., 2013b) on M. aeruginosa, $S$. obliquus existed as unicellular cell at low and high specific growth rates, but form colonies when the specific growth rate is in the range of $0.3-0.5 \mathrm{day}^{-1}$. Our results also demonstrated that the mean particle size of $S$. obliquus at low growth rate was similar to those observed at the median specific growth rate. This phenomenon revealed that the cell volume of $S$. obliquus changed at different specific growth rates.

Our results showed that volume per cell of S. obliquus decreased along with the increased of specific growth rates (Fig. 4). Chen et al. (2011) also found that cell volume of $S$. obliquus was higher at the lower temperature and phosphorus concentration. Their results indicated that cell volume of $S$. obliquus was higher at a lower specific growth rate, which confirmed our finding.

As shown in Figure 4, the maximum value of the mean cell volume $\left(469 \mu \mathrm{m}^{3}\right.$ when the specific growth rate was 0.14 day $^{-1}$ ) was almost five times higher than
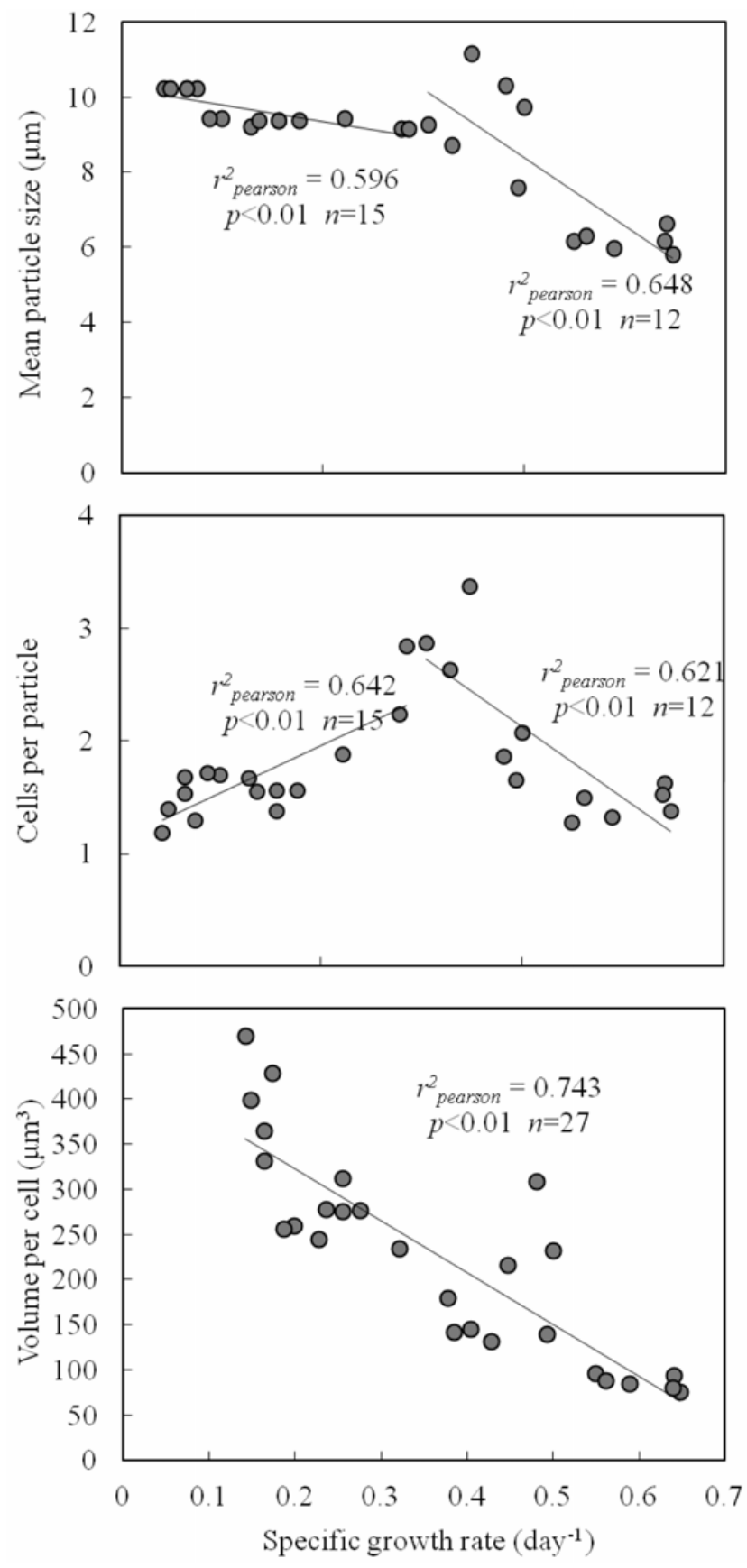

Fig. 4. Morphological characteristics of S. obliquus at different specific growth rates.

the minimum value of the mean cell volume $\left(75 \mu \mathrm{m}^{3}\right.$ when the specific growth rate was $0.64 \mathrm{day}^{-1}$ ). This result indicated that Scenedesmus cells having similar biochemical components would show different EPS content per cell because the biovolume (or dry weight) of different cells would be different. Li et al. (2013b) defined this effect caused by the variation of cellular dry weight as dilution effect. However, the maximum EPS content per cell $\left(1.36 \mathrm{pg}^{-1}\right.$ cell $\left.^{-1}\right)$ with a specific growth rate of $0.14 \mathrm{day}^{-1}$ was 11 times higher than the minimum value $\left(0.11 \mathrm{pg} \mathrm{cell}^{-1}\right)$ when the specific growth rate was $0.64 \mathrm{day}^{-1}$. The ratio of the maximum value to the 
minimum one of EPS content per cell was more than two times the ratio of biovolume (or dry weight). Thus, the dilution effect (Li et al., 2013b) caused by the variation of cell volume at different specific growth rates on cellular EPS content can be neglected here and the ratio of EPS to biomass of $S$. obliquus increased with the decrease of specific growth rate.

Although it has been well documented that colony formation of $S$. obliquus can be induced by grazer (van Holthoon et al., 2003), aquatic macrophyte Stratiotes aloides (Mulderij et al., 2005) and Potamogeton malaianus (Wu et al., 2007), glyoxylate (Liu et al., 2010) and surfactant (Lürling, 2006), the mechanism of S. obliquus colony formation was still not clear. We inferred that increasing EPS content could contribute to colony formation of $S$. obliquus based on the previous studies on C. pyrenoidosa (Yang et al., 2010) and M. aeruginosa (Liu et al., 2011). Although previous study demonstrated that the cells per particle significantly related to TPS content of S. obliquus (Liu et al., 2010), unfortunately, our results did not confirm this assumption.

Microcystis colony formation occurs via cohesion of individual cells through a structureless slimy layer called mucilage consisting predominantly of EPS (Kessel and Eloff, 1975; Plude et al., 1991). The ultrastructure of Scenedesmus showed that Scenedesmus cells joined to each other by a layer of some materials (Pickett-Heaps and Staehelin, 1975). These materials were in the gap between the continuous trilaminar sheath and the ornamented layer (Pickett-Heaps and Staehelin, 1975), consequently, they should not be considered as EPS even if the composition of this layer was EPS. Based on the above discussion, it is explained why the increasing EPS contributes to colony formation of M. aeruginosa but not to that of $S$. obliquus.

Grazer-inducing colony formation occurred in both $S$. obliquus and M. aeruginosa. However, the mechanisms of colony formation for these two species would be different. There are two main pathways of colony formation of alga (Lürling and van Donk, 1997): (i) divided cells failed to loosen (named Path 1 in the current study); (ii) adhesion of already existing single cells (named Path 2 in the current study). Colony formation of S. obliquus was generally considered by means of Path 1 because of regular cell arrangement (Lürling, 2006); by contrast, Path 2 was thought to be vest in $M$. aeruginosa due to the irregular cell arrangement (Yang and Kong, 2013). Thus, similar to S. obliquus, EPS would not be the material basis of colony formation of some alga forming colonies through Path 1. The future research on the mechanisms of colony formation of $S$. obliquus should focus on the regulation of gene expression for the layer joined cells rather than EPS.

Acknowledgements. This study was funded by the National Natural Science Foundation of China (Grant 51409216; 51309019), China Postdoctoral Science special Foundation (Grant 2015T81054) and the China Postdoctoral Science Foundation (Grant 2014M562459).

\section{References}

Agrawal A.A., 2001. Phenotypic plasticity in the interactions and evolution of species. Science, 294, 321-326.

Allen M.M., 1968. Simple conditions for growth of unicellular blue-green algae on plates. J. Phycol., 4, 1-4.

Chen M., Li J., Dai X., Sun Y. and Chen F., 2011. Effect of phosphorus and temperature on chlorophyll a contents and cell sizes of Scenedesmus obliquus and Microcystis aeruginosa. Limnology, 12, 187-192.

Chu S., 1942. The influence of the mineral composition of the medium on the growth of planktonic algae: part I. methods and culture media. J. Ecol., 30, 284-325.

Hairston N.G., Holtmeier C.L., Lampert W., Weider L.J., Post D.M., Fischer J.M., Cáceres C.E., Fox J.A. and Gaedke U., 2001. Natural selection for grazer resistance to toxic cyanobacteria: evolution of phenotypic plasticity? Evolution, 55, 2203-2214.

Kessel M. and Eloff J.N., 1975. The ultrastructure and development of the colonial sheath of Microcystis marginata. Arch. Microbiol., 106, 209-214.

Lampert W., Rothhaupt K.O. and Von Elert E., 1994. Chemical induction of colony formation in a green alga (Scenedesmus acutus) by grazers (Daphnia). Limnol. Oceanogr., 39, 1543-1550.

Li M., Zhu W., Dai X. and Li X., 2013a. Effects of linear alkylbenzene sulfonate on extracellular polysaccharide content and cells per particle of Microcystis aeruginosa and Scenedesmus obliquus. Fresen. Environ. Bull., 22, 1189-1194.

Li M., Zhu W., Gao L. and Lu L., 2013b. Changes in extracellular polysaccharide content and morphology of Microcystis aeruginosa at different specific growth rates. J. Appl. Phycol. 25, 1023-1030.

Li M., Zhu W. and Gao L., 2014. Analysis of cell concentration, volume concentration, and colony size of Microcystis via laser particle analyzer. Environ. Manage., 53, 947-958.

Liu Y., Wang W., Zhang M., Xing P. and Yang Z., 2010. PSII-efficiency, polysaccharide production, and phenotypic plasticity of Scenedesmus obliquus in response to changes in metabolic carbon flux. Biochem. Syst. Ecol. 38, 292-299.

Liu Y., Wang W., Geng L.L., Chen Y.F. and Yang Z., 2011. Polysaccharide content and morphology of Microcystis aeruginosa in response to changes in metabolic carbon flux. Fresen. Environ. Bull., 20, 1046-1050.

Lürling, M. 1999. Grazer-induced coenobial formation in clonal cultures of Scenendesmus obliquus (Chlorococcales, Chlorophyceae). J. Phycol., 35, 19-23.

Lürling M., 2003. Phenotypic plasticity in the green algae Desmodesmus and Scenedesmus with special reference to the induction of defensive morphology. Ann. Limnol. - Int. J. Lim., 39, 85-101.

Lürling M., 2006. Effects of a surfactant (FFD-6) on Scenedesmus morphology and growth under different nutrient conditions. Chemosphere, 62, 1351-1358.

Lürling M. and van Donk E., 1997. Morphological changes in Scenedesmus induced by infochemicals released in situ from zooplankton grazers. Limnol. Oceanogr., 42, 783-788.

Mulderij G., Mooij W.M. and Van Donk E., 2005. Allelopathic growth inhibition and colony formation of the green alga 
Scenedesmus obliquus by the aquatic macrophyte Stratiotes aloides. Aquat. Ecol., 39, 11-21.

Pickett-Heaps J.D. and Staehelin L.A., 1975. The ultrastructure of Scenedesmus (Chlorophyceae). II. Cell division and colony formation. J. Phycol., 11, 186-202.

Plude J.L., Parker D.L., Schommer O.J., Timmerman R.J., Hagstrom S.A., Joers J.M. and Hnasko R., 1991. Chemical characterization of polysaccharide from the slime layer of the cyanobacterium Microcystis flos-aquae C3-40. Appl. Environ. Microbiol., 57, 1696-1700.

Trainor F., 1993. Cyclomorphosis in Scenedesmus subspicatus (Chlorococcales, Chlorophyta): stimulation of colony development at low temperature. Phycologia, 32, 429-433.

van Holthoon F.L., van Beek T.A., Lürling M., Van Donk E. and De Groot A., 2003. Colony formation in Scenedesmus: a literature overview and further steps towards the chemical characterisation of the Daphnia kairomone. Hydrobiologia, 491, 241-254.

Wu Z., Deng P., Wu X., Luo S. and Gao Y., 2007. Allelopathic effects of the submerged macrophyte Potamogeton malaianus on Scenedesmus obliquus. Hydrobiologia, 592, 465-474.
Yang Z. and Kong F., 2013. Abiotic factors in colony formation: effects of nutrition and light on extracellular polysaccharide production and cell aggregates of Microcystis aeruginosa. Chin. J. Oceanol. Limnol. 31, 796-802.

Yang Z. and Li J.J., 2007. Effects of Daphnia-associated infochemicals on the morphology and growth of Scenedesmus obliquus and Microcystis aeruginosa. J. Freshwater Ecol., 22, 249-253.

Yang Z., Kong F.X., Shi X.L., Xing P. and Zhang M., 2007. Effects of Daphnia-associated infochemicals on the morphology, polysaccharides content and PSII-efficiency in Scenedesmus obliquus. Int. Rev. Hydrobiol., 92, 618-625.

Yang Z., Kong F.X., Shi X.L., Zhang M., Xing P. and Cao H.S., 2008. Changes in the morphology and polysaccharide content of Microcystis aeruginosa (Cyanobacteria) during flagellate grazing. J. Phycol. 44, 716-720.

Yang Z., Liu Y., Ge J., Wang W., Chen Y.F. and Montagnes D.J.S., 2010. Aggregate formation and polysaccharide content of Chlorella pyrenoidosa Chick (Chlorophyta) in response to simulated nutrient stress. Bioresource Technol. $101,8336-8341$. 